\title{
Interactive comment on "A 3D particle Monte Carlo approach to studying nucleation" by Christoph Köhn et al.
}

\section{Christoph Köhn et al.}

koehn@space.dtu.dk

Received and published: 22 September 2017

[Uploaded as PDF]

Please also note the supplement to this comment:

https://www.atmos-chem-phys-discuss.net/acp-2017-269/acp-2017-269-AC2-

supplement.pdf

Interactive comment on Atmos. Chem. Phys. Discuss., https://doi.org/10.5194/acp-2017-269, 2017. 